\title{
Surgical Treatment of Voluminous Jaw Cysts with a Buccal Plate: A Study of 20 Clinical Cases
}

\author{
Bertrand Baumann ${ }^{1}$, Pierre Saez ${ }^{2}$, Rémi Curien ${ }^{3}$, Marc Engels-Deutsch ${ }^{4}$
}

\begin{abstract}
Aim and objective: The aim of this paper is to present, with a series of clinical cases, some advantages of a modified surgical technique using fixed bone flaps in an approach to voluminous maxillary or mandibular odontogenic cysts (excluding keratocysts) and highlight the positive impact on the healing of mucus and bone tissues.

Background: The surgical approach of the enucleation of voluminous maxillary cysts is generally realized with a subtractive osteoplasty. The major problem with this kind of procedure is frequent fibrous healing (or scar formation) of the cavity due to mucosal invagination, especially for large lesions more than $2 \mathrm{~cm}$ in diameter. Several techniques have been proposed to limit these side effects. Very contrasting results have been observed in the techniques with graft or exogenous materials, and the scarring effects on mucus and bone tissues are poorly described. In situations where a vestibular cortical bone remains, our modification of the former technique is the use of this bone like a repositioned flap. Technique: The present study is a cases series and was carried out on 20 adult patients with maxillary or mandibular cystic lesions larger than $25 \mathrm{~mm}$. For all the patients, our modified technique consisted of using a bone flap to expose more widely the site and to carry out the enucleation of the cyst. Repositioning the flap in the final stage of the operation with osteosynthesis material allowed controlled mucosal and bone healing confirmed by clinical and radiographic follow-up.

Conclusion: In all cases of our study, no invagination of the soft tissues in the cystic cavity was observed and postoperative bone volumes were identical to the initial state. Only minor postoperative complications were observed in three cases.

Clinical significance: A bone flap approach seems to allow a tissue interception, thus better control of mucosal and bone healing, which is borne out by the clinical and radiographic controls 24 months after surgery.

Keywords: Bone flap, Cystectomy, Odontogenic cysts, Oral surgery.

The Journal of Contemporary Dental Practice (2021): 10.5005/jp-journals-10024-3184
\end{abstract}

\section{BACKGROUND}

Jaw cysts are common pathologies in the oral and maxillofacial regions. The localization of odontogenic jaw cysts is very much dependent on the origin of the cyst and their expansion depends on their preoccupied anatomic area. They are usually characterized by a small and asymptomatic growth that mostly results in large cavities. The discovery of a voluminous maxillary or mandibular cyst ( $25 \mathrm{~mm}$ or more) is generally due to the chance because it is seen on an X-ray and may compromise the initial treatment plan of the patient. A quick intervention is needed so as to limit the progression of the lesion.

The surgical approach to the enucleation of maxillary cysts is extensively described by various authors ${ }^{1,2}$ and classically cystectomy, marsupialization, decompression, and curettage are the keywords of the classical techniques used for removing large odontogenic cysts. ${ }^{3-5}$ Techniques and approaches have evolved with the progress of imaging. Three-dimensional digital reconstruction from scan acquisition ${ }^{6}$ allows us to know the precise location of cysts, thus opting for the best suited and least invasive surgical technique.

Currently, in dentistry, there is a general agreement on tissue preservation; in oral surgery, bone volume preservation is recommended as well ${ }^{7}$; maxillary surgery should meet these standards. Oral surgery should not jeopardize possible prosthetic rehabilitation with or without implant support. Treating postoperative aftereffects brought about by surgery on voluminous maxillary or mandibular cysts may sometimes require a second operation, such as bone distraction or bone graft, which allows in
${ }^{1}$ Dental Department, Central Hospital, Mulhouse, France

${ }^{2}$ ENT Department, Central Hospital, Mulhouse, France

${ }^{3}$ Service d'Odontologie, Regional Hospital of Mercy, Metz, France

Corresponding Author: Marc Engels-Deutsch, Regional Hospital of Mercy, Metz, France, Phone: +33670964217, e-mail: m.engelsdeutsch@chr-metz-thionville.fr

How to cite this article: Baumann B, Saez P, Curien R, et al. Surgical Treatment of Voluminous Jaw Cysts with a Buccal Plate: A Study of 20 Clinical Cases. J Contemp Dent Pract 2021;22(9):1069-1075.

Source of support: Nil

Conflict of interest: None

restoring the loss of bone volume. ${ }^{8}$ In these ways, the approach to the enucleation of maxillary cysts using bone flaps from the remaining cortical bone allows it to be more conservative.

The initial idea for our technique was the cut of a bone flap which was put back into place at the end of the surgery during a root resection of the mandibular molar. Many studies have proved that this approach has not only made the intervention simpler but also gave better results than root resections without bone flaps., ${ }^{9,10}$ This is the reason why this technique is increasingly used for other interventions, such as removal of foreign bodies, deep crown-root fractures, deeply embedded teeth-chiefly in the anterior region-foreign bodies in the maxillary sinus, removal of fractured and osseointegrated implants, as well as lateralization of the inferior alveolar nerve. 
The objectives of the study are to assess the advantages of the use of a bone flap to secure spontaneous bone regeneration by preventing the invagination of the soft tissues in order to allow appropriate rehabilitation.

\section{TECHNIQUe}

The present study was carried out over a period of 36 months from 2017 to 2020 on 20 adult patients (14 men and 6 women aged 40-60), all operated by the same surgeon in the Dental Department at the Central Hospital of Mulhouse (France) under general anesthesia, with maxillary or mandibular cystic lesions $25 \mathrm{~mm}$ large or more. The patients were selected after a cone-beam computed tomography $(C B C T)$ on the sole criterion of the visualization of a continuous bone all around the lesion (Fig. 1). CBCT data with radiolucency areas suspecting other lesions than cysts were excluded by the radiologist. Age, sex, or associated systemic pathologies, having no bearing on the physiology of bone tissues, were not considered in our selection process. For all cases, histopathologic sampling was done at the same time as the surgery and confirmed the presence of dentigerous or radicular cysts ${ }^{11}$ and this was not a criterion of patient selection but a medical precaution.

All the patients were followed over a 24-month period with several control visits set at specific dates. They signed an informed consent form before undergoing any procedure for collecting the records necessary for the evaluation; it granted an exemption in writing by the Central Hospital of Mulhouse, France, and the Regional Hospital of Metz, France, for some control visits.

Surgical procedure, one case is pictured (Fig. 2).

All surgical sites were infiltrated with xylocaine $(10 \mathrm{mg} / \mathrm{mL})$ and 1:100,000 adrenaline (AstraZeneca ${ }^{\circledR}$, S.A, Rueil Malmaison, France). A wide incision of the mucous membrane was made at some distance from the cystic lesion. Then, an elevation of a full-thickness mucoperiosteal flap was done. Ostectomy of the cortical bone was made under abundant irrigation with sterile physiological serum with a diamond disk (MicroSaw ${ }^{\circledR}$, Dentsply Implants, S.A., Rueil Malmaison, France) at a rotation speed of $1200 \mathrm{rpm}$. The bone flap was raised with the aid of a surgical hammer and chisels and maintained in physiological serum. After enucleation of the cyst and thorough rinsing with oral Betadine ${ }^{\circledR}$ (Meda Pharma, S.A., Paris, France), the bony lid was replaced and fixed with plates and 1.5-mm diameter screws (KLS Martin, S.A.,

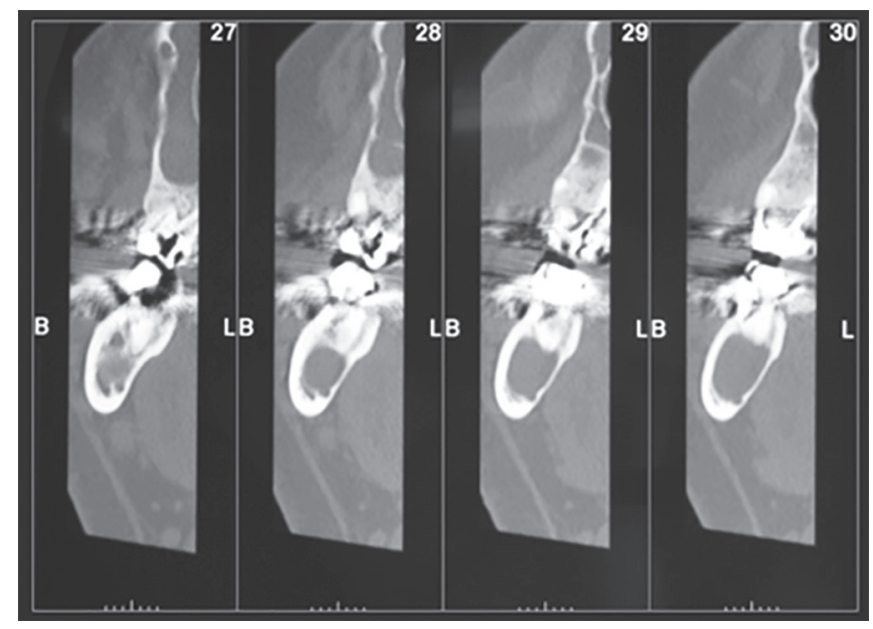

Fig. 1: Cone-beam computed tomography: Frontal views of continuous bone around a mandibular cyst located in the 46 region
Colmar, France). Repositioning of the mucous membrane was done after the release of the periosteum. Stitching was made in one or two planes depending on the tension remaining on the mucosal flaps. An extraoral adhesive compression bandage was put on the wound the first 48 hours after surgery.

All patients were prescribed the same postoperative medication without interfering with the ongoing medication or patient's initial condition: amoxicillin (Clamoxyl ${ }^{\circledR}$, GlaxoSmithKline, S.A., Marly-leRoi, France) $1 \mathrm{~g}$ three times a day with meals for 7 days; prednisolone (Solupred $^{\circledR}$, Sanofi-Aventis, S.A., Paris, France) 1 mg/kg/day with meals for 5 days; paracetamol (Doliprane ${ }^{\circledR}$, Sanofi-Aventis, S.A., Paris, France) $1 \mathrm{~g} / 6$ hours in the case of pain; and mouthwash with chlorhexidine at $0.12 \%$ (Paroex ${ }^{\circledR}$, Sunstar, S.A., Levallois Perret, France) three times a day after each meal for 15 days.

A clinical postoperative examination was made on the 7th day and $3,6,9$, and 12 months. Bone regeneration and reduction of the residual cavities and bone density were evaluated with the analysis of preoperative and postoperative panoramic radiographs and computed tomographies at 12, 18, and 24 months after surgery.

The 20 patients mentioned no postoperative pain higher than 3 in the visual analog scale the first week of healing. A postoperative swelling could be observed on average the first 3 days, being at its largest after 24 hours.

The first postoperative clinical checkup was performed after 7 days to ensure the stability of the mucosal repositioning and the nonexposure of the osteosynthesis material. The healing time of the mucous membrane ranged from 4 to 6 weeks. After 3 months, the whole site was clinically stable: keratinized gingiva adhered to underlying bone tissue and incision lines disappeared.

After 6 months, minor postoperative complications were observed in three patients: two with screw exposure and one with a fistula in the palate. For these three patients, new surgery was necessary: for the screw exposure, removal of the osteosynthesis equipment under local anesthesia was done, and for the presence of the palatal fistula, a resin palatal plate was made in the lab and then worn for 3 weeks by the patient so as to avoid sucking reflex, thus allowing healing of the mucous membrane.

For these three patients, there was a favorable outcome without any impact on surgical sites; the healing of the mucosa and reossification of sites developed in the same conditions as the other patients in this study. No wound breakdown was observed due to a too large blood clot degeneration since no grafting of any kind was carried out.

Clinically, after 12 months, the healing of the mucosa was stable: there was no invagination of the soft tissues. Postoperative volumes were back to the initial state.

For example, in the illustrated case, a panoramic X-ray was taken after 18 months. By then, it was possible to assess bone healing: the radiopacity area on the $\mathrm{X}$-ray corresponding to the initial bone lysis (Fig. 3A) seemed to be filled with a radiopaque bone, which indicated remineralization. Good ossification of the cystic cavity and reintegration of the bone flap were observed. The limits of the osteotomy are no longer visible (Fig. 3B).

\section{Discussion}

The surgical technique described above is only one stage of the overall patient treatment. It is part of a multidisciplinary treatment plan where a radiologist, dental surgeon, oral surgeon, and ENT surgeon may be required. The dental surgeon and the oral surgeon intervene simultaneously to ensure better treatment. Before 

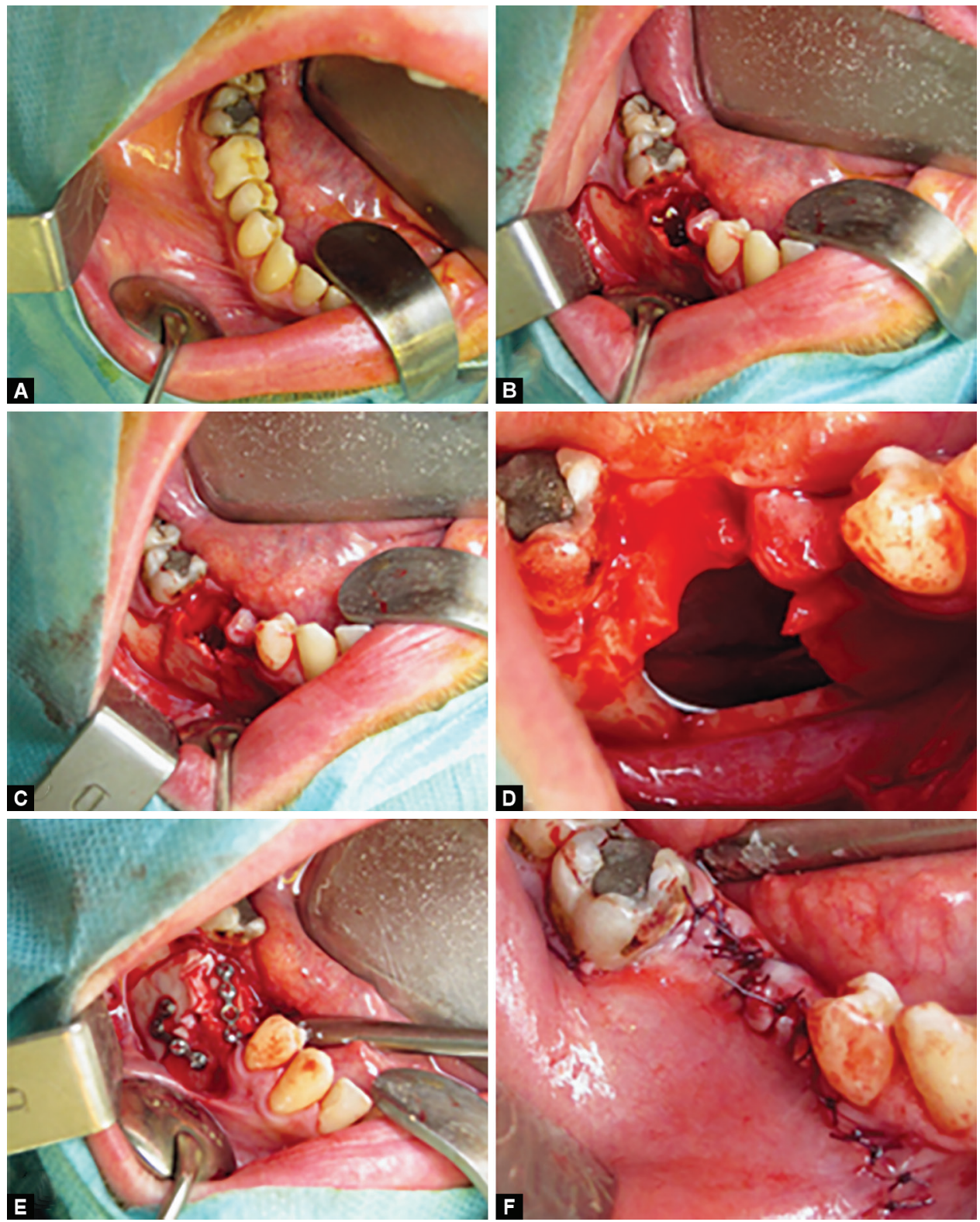

Figs $2 \mathrm{~A}$ to F: Surgical treatment of a voluminous odontogenic cyst in the 46 region: (A) Preoperative initial clinical situation; (B) Endobuccal view after extraction of 46; (C) Osteotomy of the vestibular bone using a diamond disk; (D) Enucleation of the cyst at the 46 and visibility of the alveolar nerve after excision; (E) Osteosynthesis of the vestibular bone and fixation of the bone crest; $(F)$ Repositioning of mucous membrane by stitching
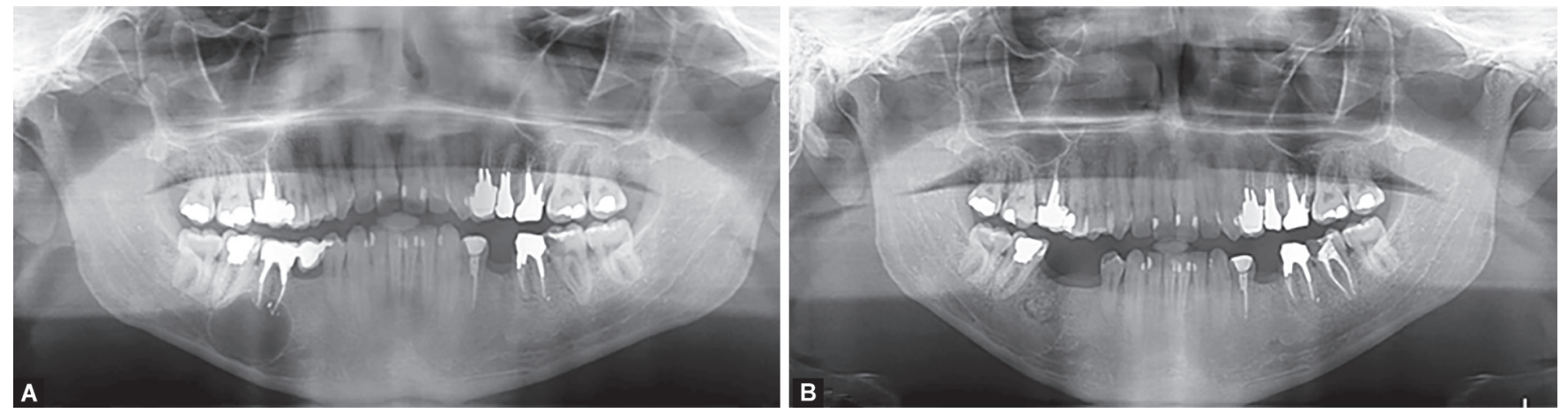

Figs 3 A and B: (A) Initial panoramic radiography: large apical cyst on 46; (B) Panoramic radiograph 18-month postprocedure, healing, and ossification 
surgery, the teeth that can be preserved within the surgical site must undergo well-sealed endodontic treatment and coronal restoration, ${ }^{12}$ and they may require retreatment to achieve the best chances of healing. ${ }^{13}$

Hospital technical facilities may be necessary to offer the best conditions for surgery: analgesia during operation and patient setup for general anesthesia allow better conditions for long-lasting surgical procedures compared to local anesthesia. The sterilization standards of a hospital operating room provide further security.

Our decision to change the surgical technique usually described was based on our response to a failed intervention. A 40-year-old female patient, sent to us for a palatal fistula by her general dentist, had undergone enucleation of a 4-cm diameter cystic lesion in the anterior maxillary region, performed using a conventional technique with a mucosal flap, subtractive osteotomy, enucleation, and repositioning of the mucosal flap and sutures. The 2D X-ray image, completed with a 3D reconstruction, taken 1 year after the surgery, showed major bone and mucous substance loss, along with missing buccal cortical plates as well as an invagination of the mucous membrane, leading to vestibular palatal continuity. This case illustrates the competition between tissues after removal of voluminous cysts in the maxillary region (Fig. 4) and further substantiates the use of bone flaps as a more appropriate surgical approach. Instead of removing the alveolar bone by milling to access the cystic lesion, we kept a bone flap which was repositioned at the end of the surgery in order to create a formwork stabilized by osteosynthesis screws as in the case of autogenous bone grafts. The interposition of a bone flap prevents soft tissue invagination and maintains good dimensional stability for the hard tissues of the surgical site useful for future implant/prosthetic rehabilitation.

As far as the surgical technique is concerned, the benefits of our protocol lied in the larger approach to the cystic lesion through the bone and better visualization of the operating field, especially when a mandibular nerve dissection is needed, ${ }^{14}$ thus reducing the risk of a nerve injury. ${ }^{15}$

In contrast to the conventional approach-subtraction osteotomy with a round bur irrigated by physiological serumwe cut a bone flap with a diamond disk: bone loss is limited to the osteotomy lines. Simple enucleation through subtraction osteotomy, ${ }^{16}$ Brosch procedure osteotomy with a wide cut of

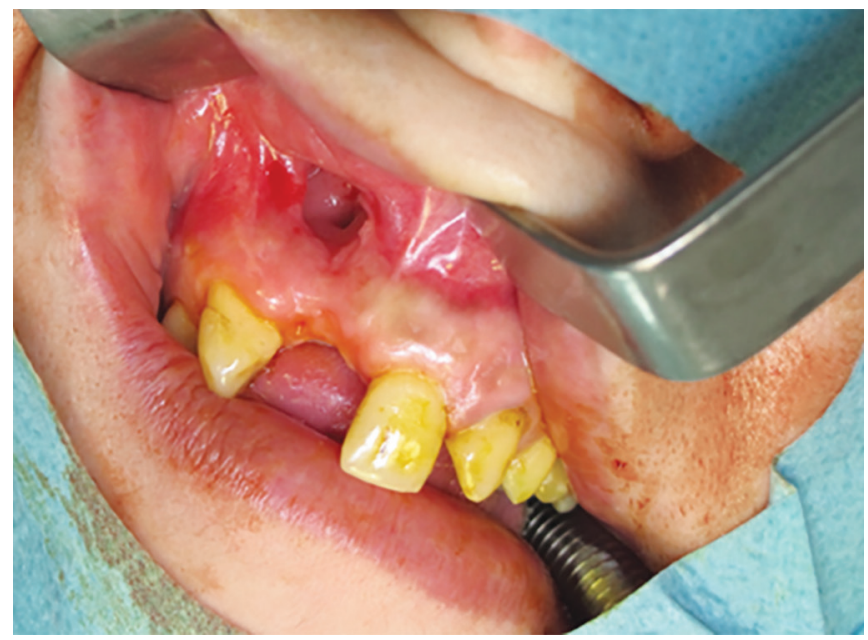

Fig. 4: Clinical view of a failed intervention in the anterior maxillary region showing an invagination of the mucous membrane after cyst enucleation without the use of a bone flap the adjacent mucosa, or osteotomy with uninterrupted "en bloc" resection of maxillaries ${ }^{17}$ causes substantial bone loss during surgery, bone resorption around the site, lack of support for soft tissues, and tissue invagination into the emptied bone cavity.

Our technique could be compared with piezosurgery in which visibility and penetration control of the osteotomy insert are benefits even if the operating time is longer. 18,19

As far as our technique could be considered conservative, the bone lid fixation with miniplates is a guarantee of bone stabilization after surgery while resorbable sutures could cause a healing hazard. ${ }^{14,20}$

Other treatments of enucleated odontogenic jaw cysts are used. As shown by Kreidler et al., autogenous cancellous bone is considered the best grafting material and has been used with clinical success for the treatment of cystic lesions for many years. ${ }^{21}$ However, donor site morbidity, either intraoral or extraoral, is a factor when obtaining a bone graft, and it is an added morbidity risk regardless of site $^{22}$ and there is an increased risk of postoperative infection. ${ }^{23}$ This reflects the need for considering all risk factors to avoid postoperative graft failure. ${ }^{24}$ The conservation of the cortical bone thanks to the bone flap removed at the outset of the surgery associated with its repositioning at the end of the operation allows us to prevent bone harvesting in another site, thereby guaranteeing better tissue preservation. It provides a physiological barrier to control healing of the mucosa and protection of the underlying trabecular bone, thus preventing any bone loss. ${ }^{9}$ By comparison, tooth extraction typically leads to loss of ridge width and height. ${ }^{25}$ The autogenous bone of the surgical site was the only source of hard tissue that comes into play in this technique. Spontaneous bone healing occurred in these large bone defects as described by Ihan Hren and Miljavec ${ }^{26}$ and without an interfering blood clot.

Filling or not filling enucleated odontogenic jaw cysts is another issue. If a tissue-engineered bone could be an alternative viable but costly filling material for cysts, ${ }^{27}$ actual reviews do not make the case for the need of filling after these kinds of surgeries. ${ }^{28,29}$

Platelet-rich plasma is sometimes used, but after removal of jaw cysts, no significant increase of bone regeneration could be observed compared with patients who did not receive this technique. $^{30}$

Exogenous materials may allow bone volume restoration as does the autogenous bone but put the patient at heightened infection risk in case of intraoral exposure and biomaterial losses. ${ }^{28,31}$

Guided tissue regeneration is also a technique used but no bone regeneration could be observed and the need for an early removal of the membranes is sometimes necessary. ${ }^{32}$

In our study, no synthetic bone replacement material, void filler, or membrane was used.

Bone remodeling and the growth factors, which contribute to it, have been extensively discussed in international studies. The osteoblastic differentiation process induced by nanostructured biomaterials is still debated to this day. ${ }^{33}$

Ostectomy through a bone flap ensures an unobstructed view of the lesion and an optimal surgical approach without any impact on healing. Comparatively, conventional ostectomy will be kept to a strict minimum to minimize as much as possible mucosa invagination. As shown by Khoury and Hensher, our approach demonstrates thus a clear advantage as far as accessibility and visibility of the surgical site are concerned. ${ }^{9}$ In the cases of good cortical bone thickness, we preferred the use of osteosynthesis 

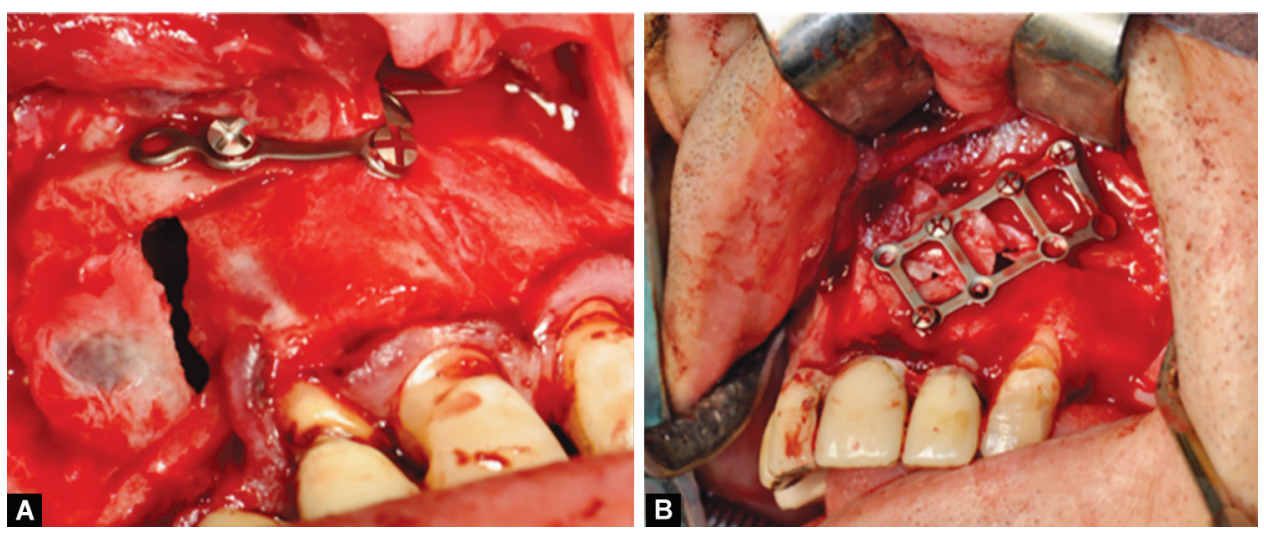

Figs 5A and B: Two cases of treatment of odontogenic cysts in the anterior region of the maxilla: (A) Use of an L-shaped plate around the anterior nasal spine; (B) Use of a scale-shaped plate to ensure tight closing of a voluminous cavity

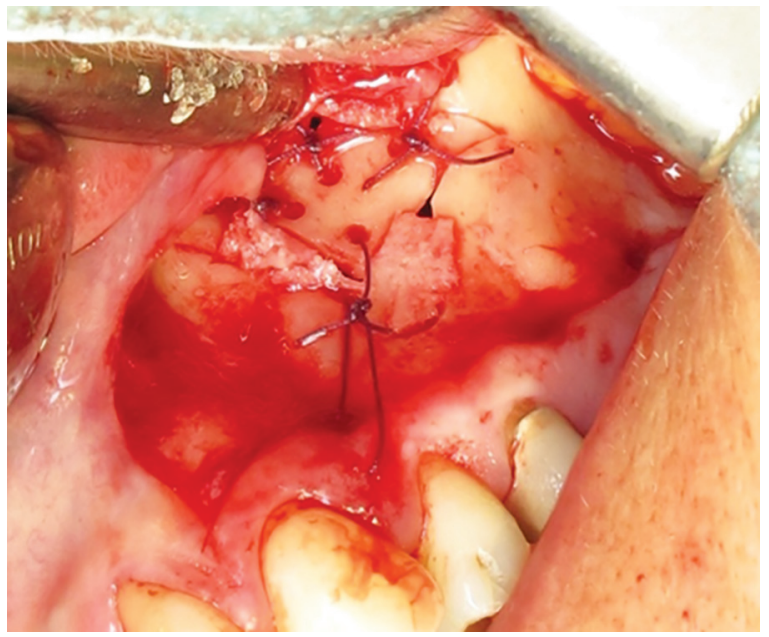

Fig. 6: Treatment of an odontogenic maxillary cyst in the 24 region, the bone flap is held with an absorbable suture material when the bone is thin

screws, and in the cases of thinnest cortical bone, we preferred stabilization of the bone flap with sutures.

Decompression or marsupialization ${ }^{34}$ are two-stage surgical procedures but also moderately invasive techniques, which aim to decrease the cyst volume before its enucleation. The use of a bone flap may also be considered at the stage as a complement to marsupialization. However, with marsupialization, the healing process is long and painful. ${ }^{35}$

Enucleation under endoscopic control has also been described by Nestal Zibo and Miller and Wang et al. as a minimally invasive surgical technique, ${ }^{35}$ preserving the surrounding tissues. ${ }^{36}$ It allows a conservative surgical approach for the bone but performing the surgery may prove difficult for practitioners unfamiliar with this technique.

The morphology of the site, the volume of the cystic lesion as well as the displacement of anatomical structures resulting from the tumor development within the maxillary or mandibular bones are all criteria to be considered when performing the osteotomy path but also as far as the osteosynthesis of the bone flap is concerned.

Depending on the dimensions of the bone flap, its thickness, the peripheral suture anchor sites, and the nearby fundamental anatomical zones, the osteosynthesis processes can vary; the design and shaping of the plates will allow adjustment of the bone flap so as to better fit the morphology of the site. Thus, we have sometimes chosen an L-shaped plate to work around the anterior nasal spine (Fig. 5A) or a scale-shaped one which allowed osteosynthesis of the various fragments of the bone flap and ensured tight closing of the voluminous cavity (Fig. 5B). In one case, the bone flap was held in place with an absorbable suture material, thus avoiding another intervention to remove the plates (Fig. 6). This intra-sample variability underlined the scope of surgical possibilities of this technique as well as the wide range of therapeutic applications.

Edema was observed on average for 3 days with a peak on the first day. When the surgery took longer, the duration of the inflammation was protracted in some patients; both were then correlated. ${ }^{37}$ The edema was also larger when osteosynthesis miniplates were used compared to fixation with screws only. ${ }^{38}$

During prosthetic rehabilitation, three patients had to be operated on again to remove the osteosynthesis material. In the course of these interventions, complete cortical healing without a bone loss was observed and implant placement was possible.

Spontaneous bone regeneration after enucleation of large jaw cysts without the aid of any filling materials could be assessed by postoperative panoramic radiographs and computed tomography. ${ }^{39-41}$ Our radiographic examination 12 , 18 , and 24 months after surgery allowed visualization of bone remineralization and trabeculation. Reossification of the site was observed in all patients. The radiolucency right after surgery had completely opacified after a year. The quality of the newly formed bone tissues on the surgical site looked identical to the adjacent healthy areas: radiopacity, bone structure, as well as the degree of mineralization were comparable. The fact that there was no bone substance loss after surgery allowed prosthetic rehabilitation in good conditions for all patients. All the prosthetic restorations whether they are without implants for removable prostheses or tooth- and implant-supported in fixed prostheses were possible in the same conditions as before surgery.

\section{Conclusion}

The surgical treatment of voluminous maxillary or mandibular cysts may now be undertaken without bone loss. The repositioning of the bone flap with proper stabilization acts as a biological barrier; it controls bone healing and prevents the commonly observed mucosal invagination. Our case series show that spontaneous 
bone regeneration can occur after this surgical removal of jaw cysts without the aid of any graft materials in the large cystic cavity sufficiently surrounded by enough bony walls, as seen elsewhere ${ }^{42}$ even if they are thin.

\section{Clinical Significance}

The use of a bone flap allows a conservative approach to hard tissues without affecting the mucosa. Not using fillings, grafts, or exogenous materials simplifies the surgical procedure and decreases the overall cost of surgery.

Thus, surgery becomes a step in the global treatment plan and not an obstacle, which may get in the way of prosthetic rehabilitation. Intervention on bone and mucosal tissues may be undertaken in the early stage without compromising the following treatment. This clinical follow-up of 20 cases reveals encouraging results without complicating postoperative care procedures.

\section{References}

1. Morgan TA, Burton CC, Qian F. A retrospective review of treatment of the odontogenic keratocyst. J Oral Maxillofac Surg Off J Am Assoc Oral Maxillofac Surg 2005;63(5):635-639. DOI: 10.1016/ j.joms.2004.07.026.

2. Fleury JE, Deboets $D$, Maffre N, et al. [Dentigerous cysts: apropos of 40 cases]. Rev Stomatol Chir Maxillofac 1994;95(2):87-90. PMID: 8036439.

3. Bonavolontà $P$, Dell'Aversana Orabona G, Friscia M, et al. Surgical management of large odontogenic cysts of the mandible. J Craniofac Surg 2019;30(7):e658-e661. DOI: 10.1097/SCS.0000000000005725.

4. Soliman MM, El Dayem Hassan HA, Elgazaerly H, et al. Marsupialization as a treatment modality of large jaw cysts. World Appl Sci J 2013;21(12):1752-1759. DOI: 10.5829/idosi.wasj.2013.21.12.99.

5. Yüzügüllü B, Araz K. Validity of conventional surgical treatment methods for mandibular dentigerous cysts. Two case reports. N Y State Dent J 2011;77(2):36-39. PMID: 21678870.

6. Orentlicher G, Goldsmith D, Horowitz A. Applications of 3-dimensional virtual computerized tomography technology in oral and maxillofacial surgery: current therapy. J Oral Maxillofac Surg Off J Am Assoc Oral Maxillofac Surg 2010;68(8):1933-1959. DOI: 10.1016/j.joms.2010.03.013.

7. Levin L, Zigdon H, Mayer Y. [Alveolar ridge preservation following tooth extraction]. Refuat Ha-Peh Veha-Shinayim 1993 2008;25(1): 41-46, 83. PMID: 18661801.

8. Rachmiel A, Emodi O, Aizenbud D. [Reconstruction of the alveolar ridge by osteodistraction for implant placement]. Refuat Ha-Peh Veha-Shinayim 1993 2011;28(3):30-36, 69. PMID: 21939103.

9. Khoury F, Hensher R. The bony lid approach for the apical root resection of lower molars. Int J Oral Maxillofac Surg 1987;16(2): 166-170. DOI: 10.1016/s0901-5027(87)80125-x.

10. Schmidt J. [Procedures with Khoury's bone cover method for mandibular molar root resection in everyday practice]. Quintessenz 1990;41(8):1263-1270. PMID: 2217699.

11. El-Naggar AK, Chan JKC, Takata T, et al. The fourth edition of the head and neck World Health Organization blue book: editors' perspectives. Hum Pathol 2017;66:10-12. DOI: 10.1016/j.humpath.2017.05.014.

12. Zuolo ML, Ferreira MO, Gutmann JL. Prognosis in periradicular surgery: a clinical prospective study. Int Endod J 2000;33(2):91-98. DOI: 10.1046/j.1365-2591.2000.00263.x.

13. Friedman S. Retrograde approaches in endodontic therapy. Endod Dent Traumatol 1991;7(3):97-107. DOI: 10.1111/j.1600-9657.1991. tb00192.x.

14. Akçay H, Tatar B, Kuru K, et al. Bone flap technique with piezosurgery for impacted teeth extraction and bone cysts removal without additional fixation. J Craniofac Surg 2019;30(1):e21-e24. DOI: 10.1097/ SCS.0000000000004913.
15. Saponaro G, Pelo $S$, De Angelis $P$, et al. Bone flap technique for impacted teeth extraction and bone cysts removal. J Craniofac Surg 2016;27(4):1084-1086. DOI: 10.1097/SCS.0000000000002606.

16. Scharffetter K, Balz-Herrmann C, Lagrange W, et al. Proliferation kinetics-study of the growth of keratocysts. Morpho-functional explanation for recurrences. J Cranio-Maxillo-fac Surg Off Publ Eur Assoc Cranio-Maxillo-fac Surg 1989;17(5):226-233. DOI: 10.1016/ s1010-5182(89)80074-5.

17. Farmand $M$, Makek $M$. Late results following the Brosch-procedure for treating large mandibular ramus cysts. J Maxillofac Surg 1983;11(5):211-215. DOI: 10.1016/s0301-0503(83)80051-4.

18. Pappalardo S, Guarnieri R. Randomized clinical study comparing piezosurgery and conventional rotatory surgery in mandibular cyst enucleation. J Cranio-Maxillo-fac Surg Off Publ Eur Assoc CranioMaxillo-fac Surg 2014;42(5):e80-e85. DOI: 10.1016/j.jcms.2013.06.013.

19. Yaman Z, Suer BT. Clinical comparison of ultrasonic surgery and conventional surgical techniques for enucleating jaw cysts. Int J Oral Maxillofac Surg 2013;42(11):1462-1468. DOI: 10.1016/ j.ijom.2013.05.003.

20. Sivolella S, Brunello G, Fistarol F, et al. The bone lid technique in oral surgery: a case series study. Int J Oral Maxillofac Surg 2017;46(11):1490-1496. DOI: 10.1016/j.ijom.2017.06.027.

21. Kreidler JF, Raubenheimer EJ, van Heerden WF. A retrospective analysis of 367 cystic lesions of the jaw-the UIm experience. J Cranio-Maxillo-fac Surg Off Publ Eur Assoc Cranio-Maxillo-fac Surg 1993;21(8):339-341. DOI: 10.1016/s1010-5182(05)80494-9.

22. Younger EM, Chapman MW. Morbidity at bone graft donor sites. J Orthop Trauma 1989;3(3):192-195. DOI: 10.1097/00005131-19 8909000-00002.

23. Lee H, Lee S-J, Seo B-M. Investigation of postoperative complications of intrabony cystic lesions in the oral and maxillofacial region. J Oral Maxillofac Surg Off J Am Assoc Oral Maxillofac Surg 2019;77(9): 1823-1831. DOI: 10.1016/j.joms.2019.03.022.

24. Lim H-K, Kim J-W, Lee U-L, et al. Risk factor analysis of graft failure with concomitant cyst enucleation of the jaw bone: a retrospective multicenter study. J Oral Maxillofac Surg Off J Am Assoc Oral Maxillofac Surg 2017;75(8):1668-1678. DOI: 10.1016/j.joms.2017.02.003.

25. Iasella JM, Greenwell $\mathrm{H}$, Miller RL, et al. Ridge preservation with freeze-dried bone allograft and a collagen membrane compared to extraction alone for implant site development: a clinical and histologic study in humans. J Periodontol 2003;74(7):990-999. DOI: 10.1902/jop.2003.74.7.990.

26. Ihan Hren N, Miljavec M. Spontaneous bone healing of the large bone defects in the mandible. Int J Oral Maxillofac Surg 2008;37(12): 1111-1116. DOI: 10.1016/j.ijom.2008.07.008.

27. Pradel W, Eckelt U, Lauer G. Bone regeneration after enucleation of mandibular cysts: comparing autogenous grafts from tissueengineered bone and iliac bone. Oral Surg Oral Med Oral Pathol Oral Radiol Endod 2006;101(3):285-290. DOI: 10.1016/j.tripleo.2005.06.001.

28. Ettl T, Gosau M, Sader R, et al. Jaw cysts - filling or no filling after enucleation? A review. J Cranio-Maxillo-fac Surg Off Publ Eur Assoc Cranio-Maxillo-fac Surg 2012;40(6):485-493. DOI: 10.1016/ j.jcms.2011.07.023.

29. Buchbender M, Neukam FW, Lutz R, et al. Treatment of enucleated odontogenic jaw cysts: a systematic review. Oral Surg Oral Med Oral Pathol Oral Radiol 2018;125(5):399-406. DOI: 10.1016/ j.oooo.2017.12.010.

30. Ramanathan A, Cariappa KM. Effect of platelet-rich plasma on bone regeneration after removal of cysts and benign tumours of the jaws. Oral Maxillofac Surg 2014;18(4):445-452. DOI: 10.1007/s10006-013-0 435-0.

31. Merli M, Moscatelli M, Mariotti G, et al. Autogenous bone versus deproteinised bovine bone matrix in 1-stage lateral sinus floor elevation in the severely atrophied maxilla: a randomised controlled trial. Eur J Oral Implantol 2013;6(1):27-37. PMID: 23513200.

32. Buser $D$, Brägger U, Lang NP, et al. Regeneration and enlargement of jaw bone using guided tissue regeneration. Clin Oral Implants Res 1990;1(1):22-32. DOI: 10.1034/j.1600-0501.1990.010104.x. 
33. Ozdemir T, Higgins AM, Brown JL. Osteoinductive biomaterial geometries for bone regenerative engineering. Curr Pharm Des 2013;19(19):3446-3455. DOI: 10.2174/1381612811319190010.

34. Cranin AN, Madan S, Fayans E. Novel method of treating large cysts of jaws in children. NY State Dent J 1994;60(2):41-44. PMID: 8139822.

35. Nestal Zibo H, Miller E. Endoscopically assisted enucleation of a large mandibular periapical cyst. Stomatologija 2011;13(4):128-131. PMID: 22362340.

36. Wang Y, Chang S, Lin Z, et al. Endoscopic-assisted enucleation of large mandibular odontogenic cysts. Oral Surg Oral Med Oral Pathol Oral Radiol 2020;129(2):115-119. DOI: 10.1016/j.00oo.2019.05.012.

37. Penarrocha M, Garcia B, Marti E, et al. Pain and inflammation after periapical surgery in 60 patients. J Oral Maxillofac Surg Off J Am Assoc Oral Maxillofac Surg 2006;64(3):429-433. DOI: 10.1016/ j.joms.2005.11.014.

38. Agnihotri A, Prabhu S, Thomas S. A comparative analysis of the efficacy of cortical screws as lag screws and miniplates for internal fixation of mandibular symphyseal region fractures: a randomized prospective study. Int J Oral Maxillofac Surg 2014;43(1):22-28. DOI: 10.1016/j.ijom.2013.07.001.

39. Chiapasco M, Rossi A, Motta JJ, et al. Spontaneous bone regeneration after enucleation of large mandibular cysts: a radiographic computed analysis of 27 consecutive cases. J Oral Maxillofac Surg Off J Am Assoc Oral Maxillofac Surg 2000;58(9):942-948; discussion 949. DOI: 10.1053/joms.2000.8732.

40. Wagdargi SS, Rai KK, Arunkumar KV, et al. Evaluation of spontaneous bone regeneration after enucleation of large cysts of the jaws using radiographic computed software. J Contemp Dent Pract 2016;17(6):489-495. DOI: 10.5005/jp-journals-10024-1878.

41. Bodner L, Bar-Ziv J. Characteristics of bone formation following marsupialization of jaw cysts. Dento Maxillo Facial Radiol 1998;27(3):166-171. DOI: 10.1038/sj/dmfr/4600344.

42. Chacko R, Kumar S, Paul A, et al. Spontaneous bone regeneration after enucleation of large jaw cysts: a digital radiographic analysis of 44 consecutive cases. J Clin Diagn Res 2015;9(9):ZC84-ZC89. DOI: $10.7860 / J C D R / 2015 / 13394.6524$. 